\title{
Stimulation of Dorsolateral Prefrontal Cortex Enhances Adaptive Cognitive Control: A High-Definition Transcranial Direct Current Stimulation Study
}

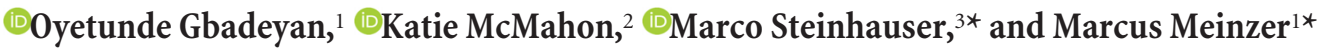 \\ ${ }^{1}$ Centre for Clinical Research, The University of Queensland, Brisbane 4029, Australia, ${ }^{2}$ Centre for Advanced Imaging, The University of Queensland, \\ Brisbane 4072, Australia, and ${ }^{3}$ Department of Psychology, Catholic University of Eichstätt-Ingolstadt, Eichstätt 85072, Germany
}

Conflict adaptation is a hallmark effect of adaptive cognitive control and refers to the adjustment of control to the level of previously experienced conflict. Conflict monitoring theory assumes that the dorsolateral prefrontal cortex (DLPFC) is causally involved in this adjustment. However, to date, evidence in humans is predominantly correlational, and heterogeneous with respect to the lateralization of control in the DLPFC. We used high-definition transcranial direct current stimulation (HD-tDCS), which allows for more focal current delivery than conventional tDCS, to clarify the causal involvement of the DLPFC in conflict adaptation. Specifically, we investigated the regional specificity and lateralization of potential beneficial stimulation effects on conflict adaptation during a visual flanker task. One hundred twenty healthy participants were assigned to four HD-tDCS conditions: left or right DLPFC or left or right primary motor cortex (M1). Each group underwent both active and sham HD-tDCS in crossover, double-blind designs. We obtained a sizeable conflict adaptation effect (measured as the modulation of the flanker effect as a function of previous response conflict) in all groups and conditions. However, this effect was larger under active HD-tDCS than under sham stimulation in both DLPFC groups. In contrast, active stimulation had no effect on conflict adaptation in the M1 groups. In sum, the present results indicate that the DLPFC plays a causal role in adaptive cognitive control, but that the involvement of DLPFC in control is not restricted to the left or right hemisphere. Moreover, our study confirms the potential of HD-tDCS to modulate cognition in a regionally specific manner.

Key words: brain stimulation; cognitive control; conflict adaptation; conflict monitoring theory; dorsolateral prefrontal cortex; highdefinition transcranial direct current stimulation

Significance Statement

Conflict adaptation is a hallmark effect of adaptive cognitive control. While animal studies have suggested causal involvement of the DLPFC in this phenomenon, such evidence is currently lacking in humans. The present study used high-definition transcranial direct current stimulation (HD-tDCS) to demonstrate that the DLPFC is causally involved in conflict adaptation in humans. Our study confirms a central claim of conflict monitoring theory, which up to now has predominantly relied on correlational studies. Our results further indicate an equal involvement of the left and right DLPFC in adaptive control, whereas stimulation of a control region - the primary motor cortex - had no effect on adaptive control. The study thus confirms the potential of HD-tDCS to modulate cognition in a regionally specific manner.

\section{Introduction}

A remarkable feature of human cognition is the ability to achieve flexible and goal-directed behavior by selectively attending to

\footnotetext{
Received Aug. 1, 2016; revised 0ct. 7, 2016; accepted 0ct. 18, 2016.

Author contributions: 0.G., K.M., M.S., and M.M. designed research; 0.G. performed research; M.S. contributed unpublished reagents/analytic tools; 0. G., M.S., and M.M. analyzed data; 0. G., K.M., M.S., and M.M. wrote the paper.

This work was supported by Australian Research Council Grant FT120100608.

${ }^{*}$ M.S. and M.M contributed equally to this work.

The authors declare no competing financial interests.

Correspondence should be addressed to Marcus Meinzer, Centre for Clinical Research, The University of Queens-

land, Brisbane 4029, Australia. E-mail:m.meinzer@uq.edu.au.
}

goal-relevant information while suppressing distracting and irrelevant information. These adaptive processes are collectively referred to as cognitive control (Miller, 2000; Botvinick et al., 2001). A predominant model of cognitive control, the conflict monitoring theory (Botvinick et al., 2001), asserts that the anterior cingulate cortex (ACC) is engaged during conflict detection and automatically triggers control processes subserved by the dorsolateral prefrontal cortex (DLPFC; Miller and Cohen, 2001; 
Kerns et al., 2004). Previous neuroimaging research in humans and lesion studies in nonhuman primates have lent substantial support for this theory. However, research in humans has mainly focused on conflict detection in the ACC. Fewer studies have investigated the consequences of conflict detection, i.e., the brain structures involved in attentional adjustments (for review, see Mansouri et al., 2009).

The original conflict monitoring theory assumes that the DLPFC is causally involved in these adjustments, but evidence for such a causal involvement has been provided only in animal studies (Mansouri et al., 2007). For humans, the available evidence is predominantly based on correlational data. Moreover, there is contradicting and heterogeneous evidence regarding the lateralization of control in the DLPFC. For example, previous imaging studies suggested that conflict adaptation (CA) may be associated with activity in the right (Kerns et al., 2004; Egner and Hirsch, 2005; Egner et al., 2008), left (MacDonald et al., 2000; Kim et al., 2014), or bilateral DLPFC (van Veen and Carter, 2005).

In the present study, we aimed to clarify the causal role and regional specificity of the left and right DLPFC involvement in CA by using high-definition transcranial direct current stimulation (HD-tDCS; Alam et al., 2016). HD-tDCS allows modulating cortical excitability and behavioral performance by administering an electrical current with superior spatial precision compared to conventional tDCS (Kuo et al., 2013; Villamar et al., 2013; Bortoletto et al., 2016). Please note, previous tDCS studies investigating effects of DLPFC stimulation on response inhibition and other forms of cognitive control (Vanderhasselt et al., 2013; Plewnia et al., 2015; Mansouri et al., 2016; Zmigrod et al., 2016) used "conventional" stimulation protocols, which do not allow to administer the current in a regionally specific way (Alam et al., 2016).

We used a visual flanker task and participants were required to respond to a centrally presented target while ignoring surrounding "distractor" stimuli that were associated with the same (congruent) or a different (incongruent) response type. Responses are typically slower for incongruent compared to congruent trials ("flanker effect," Eriksen and Eriksen, 1974). However, response latency and accuracy on a given trial are also modulated by the level of conflict on directly preceding trials. Specifically, flanker effects following incongruent trials are reduced relative to those following congruent trials, an effect known as the "conflict adaptation effect" (Gratton et al., 1992; Botvinick et al., 2001). CA is thought to reflect the flexible adaptation of control to anticipated levels of conflict (Botvinick et al., 2001) and is thus a direct measure of adaptive cognitive control. To investigate the presumed role of the DLPFC in CA, we used a placebo ("sham") HD-tDCS controlled, double-blind design and active HD-tDCS was administered during the task either to the left or right DLPFC. Two additional groups received active HD-tDCS to the left and right primary motor cortex (M1), which assessed the specificity of potential prefrontal HD-tDCS effects by stimulating regions not implicated in CA. Based on previous imaging studies that demonstrated either left or right or bilateral DLPFC involvement in CA (Kerns et al., 2004; Egner and Hirsch, 2005; van Veen and Carter, 2005; Kim et al., 2014), we hypothesized that excitatory anodal HD-tDCS administered to both the left or right DLPFC would enhance CA. Because neither the left nor right M1 have been implicated in CA in those previous studies, we hypothesized that stimulation of those control regions would not modulate performance.

\section{Materials and Methods}

Study overview and experimental design. One hundred twenty healthy right-handed participants (59 men, 61 women; mean \pm SD years, $26.02 \pm 4.69)$ were recruited for this study and assigned to four stimulation conditions: left and right DLPFC and M1 HD-tDCS $(N=30$ per condition). Each group underwent both active (i.e., excitatory "anodal" HD-tDCS, which is typically used to enhance performance; Coffman et al., 2014; Dedoncker et al., 2016) and sham HD-tDCS in a crossover, double-blind design while completing a visual flanker task to assess potential stimulation effects on behavioral performance. Potential adverse effects and blinding efficacy were also assessed in a systematic fashion. Stimulation order was counterbalanced in all groups and sessions were scheduled at least three days apart to prevent carryover effects. All experimental sessions were performed between 9 A.M. and 4 P.M. according to individual preferences, with the time of day being constant across sessions for each participant. Written informed consent was obtained before study inclusion and participants were compensated with AUD $\$ 50$. The experiment was approved by the ethics committee of the University of Queensland.

Participants. The four participant groups were matched for sex $\left(\chi^{2}=\right.$ $1.17, p=0.76$; right and left DLPFC, 15 and 17 males; right and left M1, 13 and 14 males, respectively) and age $\left(F_{(3,116)}=0.65, p=0.58\right.$; mean \pm SD years, right and left DLPFC, $26.60 \pm 4.52$ and $25.96 \pm 5.10$; right and left M1, $25.06 \pm 4.34$ and $26.46 \pm 4.48$, respectively). Participants with metal implants in the head, a cardiac pacemaker, current pregnancy, a history of seizures or frequent migraines, and chronic or acute neurologic or psychiatric disorders were excluded from the study. None of the participants reported taking any medication (e.g., antidepressants, antipsychotics, anxiolytics, anticonvulsants) or recreational drugs that may interact with tDCS effects (Stagg and Nitsche, 2011).

Behavioral task. The design of the visual flanker task was identical to that of a previous study by our group (Gbadeyan et al., 2016). The main goal of this previous study was to compare safety, blinding efficacy, behavioral effects, and MRI compatibility of conventional and highdefinition tDCS. This previous study also compared the behavioral effects of one of the groups reported in the present manuscript (right DLPFC stimulation group) with those of a group that received conventional tDCS administered to the right DLPFC. However, this study used only one HD-tDCS setup, which did not allow for investigating laterality effects or regional specificity of potential beneficial stimulation effects across different HD-tDCS setups.

In the present study, conflict was experimentally induced by a visual flanker task. During each trial, a fixation cross was presented for $300 \mathrm{~ms}$, followed by the presentation of four flanker arrows (i.e., $\ll \ll$ or $\gg \gg$ ) for $100 \mathrm{~ms}$. The target center arrow was subsequently presented for 100 $\mathrm{ms}$ together with the flanker arrows. The flanker and target arrows pointed either in the same (congruent condition, $\ll<\ll$ or $\gg>\gg$ ) or opposite directions (incongruent condition, $\ll>\ll$ or $\gg<\gg$ ). Stimuli (white arrows against a black background) were presented using e-Prime version 2.0 (Psychology Software Tools) on a 22 inch computer monitor. Participants indicated the direction of the central arrow by pressing the left or right response key as quickly and accurately as possible. On error trials, an error message was displayed for $100 \mathrm{~ms}$. A black screen was displayed for $500 \mathrm{~ms}$ until the next trial commenced. Before the first task block, participants completed a practice block of 16 trials. The task itself consisted of five blocks ( $N=240$ trials/block). The different trial types (congruent/incongruent, arrows left/right) were pseudorandomized and counterbalanced. After each block a short break was interspersed and mean accuracy and response time were displayed.

$H D$ - $t D C S$. The stimulation was administered using a one-channel direct current stimulator (DC-Stimulator Plus, NeuroConn) and two concentric rubber electrodes (Bortoletto et al., 2016; Gbadeyan et al., 2016): a small center anode (diameter, $2.5 \mathrm{~cm}$ ) and ring-shaped return cathode (inner diameter, $9.2 \mathrm{~cm}$; outer diameter, $11.5 \mathrm{~cm}$ ). This setup is a variation of the frequently used " $4 \times 1$ " HD-tDCS setup, which constrains the current flow by using four return electrodes that are arranged in a circle around a center electrode (Kuo et al., 2013; Villamar et al., 2013; Alam et al., 2016; Hogeveen et al., 2016). Safety, effective behavioral modulation, 
and focal current delivery have been demonstrated for both " $4 \times 1$ " and concentric HD-tDCS setups, but the latter was chosen because it does not require an expensive multi-channel stimulator (Bortoletto et al., 2016; Gbadeyan et al., 2016). Electrodes were attached over the target regions using an adhesive conductive gel (Weaver Ten20 conductive paste) and held in place with an EEG cap to ensure a stable conductive adhesion with the skin. The position of the center electrode (anode) was determined using the 10-20 international EEG system (left and right DLPFC, F3 and F4; left and right M1, C3 and C4, respectively). The ring cathode was positioned symmetrically around the center electrode.

In both active and sham stimulation conditions, the current was ramped up to $1 \mathrm{~mA}$ over $10 \mathrm{~s}$ before commencement of the experiment. Active HD-tDCS was administered for 20 min before ramping down. During sham tDCS, the current was ramped down after $10 \mathrm{~s}$, which elicits a physical sensation on the scalp to assure blinding of participants but does not modulate neural function. Blinding of researchers was achieved by using the "study mode" of the DC stimulator (i.e., a preassigned code triggered the respective stimulation conditions).

Adverse effects and blinding. Nine potential adverse effects (AEs; for details, see Table 3 ) were rated by all participants using a questionnaire (1, absent; 2 , mild; 3 , moderate; 4 , severe; Brunoni et al., 2011) that was administered after each stimulation session. AEs were compared between sham and anodal HD-tDCS within and between groups. The blinded investigator guessed the order of the stimulation conditions based on skin irritation (assessed on an 11-point scale; 0, no skin redness; 10, severe skin redness) after each stimulation session. Participants were asked to guess the order of stimulation immediately following the completion of the second stimulation session to assess blinding.

Statistical analyses. For the flanker task data, all analyses were conducted on mean response times (RTs) and mean error rates, computed separately for each stimulation condition and participant. To control for outliers in RT data, trials with RTs deviating more than four SDs from condition means were excluded ( $<1 \%$ of trials). Error rates were analyzed using arcsine transformed data (Winer et al., 1991). SEs of withinsubject effects were calculated using the method of Cousineau (2005).

Mean RT and mean error rates were first subjected to a five-way mixed-model ANOVA with between-subject variables Region (DLPFC, M1) and Laterality (left, right), and within-subjects variables Stimulation (active, sham), Previous Congruency (congruent, incongruent), and Current Congruency (congruent, incongruent). For brevity, we report only effects that are not qualified by higher interactions (with the exception of the overall conflict adaptation effect) in this and all following analyses. To account for region-specific effects, mean RTs were subsequently analyzed separately for the DLPFC and M1 groups using fourway mixed-model ANOVAs with the variables Laterality, Stimulation, Previous Congruency, and Current Congruency. Moreover, three-way mixed-model ANOVAs with the variables Laterality, Stimulation, and Current Congruency were conducted for mean RTs of the DLPFC groups, separately for trials following congruent and incongruent trials, to investigate whether stimulation effects on conflict adaptation are solely caused by the effects of previous congruent or incongruent trials.

Three control analyses were run to investigate potential mediating effects of additional variables on our data. First, a six-way ANOVA was conducted by including the additional variable Response Repetition (repetition, switch) in the aforementioned five-way ANOVA to investigate whether our effects are related to repetition priming (Mayr et al., 2003). Second, another six-way ANOVA was run including the variable Block (1-5) to investigate whether stimulation effects changed over the course of the experiment. Finally, the M1 groups were analyzed in a five-way ANOVA including the additional variable Response Laterality (left versus right hand response) in the aforementioned four-way ANOVAs to investigate whether stimulating the primary motor cortex influences performance for responses executed with the contralateral response hand.

Adverse effects were compared between active and sham stimulation conditions using paired $t$ tests. Furthermore, differences in adverse effects between active and sham stimulation between all four stimulation groups (left/ right DLPFC or M1) were calculated and compared using unpaired $t$ tests.
Table 1. Mean response times (in milliseconds) as a function of Stimulation (active, sham), Previous Congruency (congruent, incongruent), and Current Congruency (congruent, incongruent) in each of the four groups (left DLPFC, right DLPFC, left $\mathrm{M1}$, and right M1)

\begin{tabular}{lllll}
\hline & $c C$ & cl & iC & il \\
\hline $\begin{array}{llll}\text { Left DLPFC } \\
\text { Active }\end{array}$ & $233( \pm 2)$ & $316( \pm 3)$ & $247( \pm 2)$ & $308( \pm 3)$ \\
$\quad$ Sham & $237( \pm 3)$ & $317( \pm 3)$ & $249( \pm 3)$ & $312( \pm 3)$ \\
$\begin{array}{l}\text { Right DLPFC } \\
\text { Active }\end{array}$ & $265( \pm 4)$ & $346( \pm 4)$ & $278( \pm 4)$ & $340( \pm 4)$ \\
$\quad$ Sham & $268( \pm 3)$ & $344( \pm 5)$ & $277( \pm 3)$ & $340( \pm 5)$ \\
Left M1 & & & & \\
Active & $251( \pm 4)$ & $340( \pm 5)$ & $267( \pm 4)$ & $332( \pm 5)$ \\
Sham & $247( \pm 4)$ & $340( \pm 6)$ & $261( \pm 4)$ & $331( \pm 5)$ \\
Right M1 & & & & \\
Active & $234( \pm 4)$ & $333( \pm 6)$ & $247( \pm 4)$ & $324( \pm 5)$ \\
Sham & $233( \pm 4)$ & $331( \pm 6)$ & $248( \pm 4)$ & $320( \pm 5)$ \\
\hline
\end{tabular}

CC, Previous congruent, current congruent; $\mathrm{Cl}$, previous congruent, current incongruent; iC, previous incongruent, current congruent; il, previous incongruent, current incongruent. Values in brackets are within-subject SEMs.
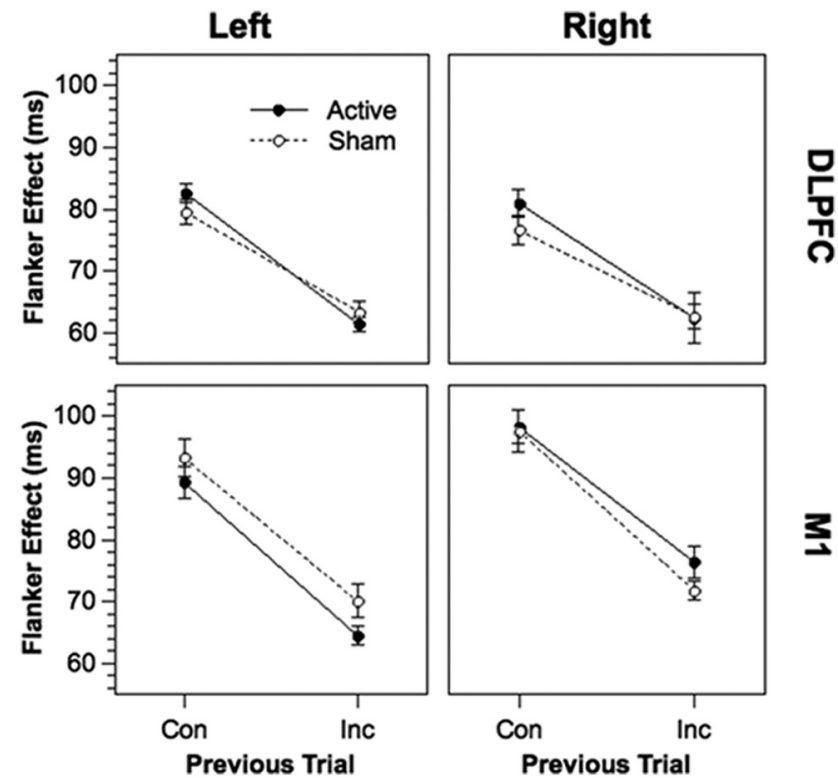

Figure 1. Flanker effects as a function of Previous Trial (congruent, incongruent) and Stimulation (active, sham) in each of the four groups (left DLPFC, right DLPFC, left M1, right M1). Slopes reflect the size of conflict adaptation in each condition. ms, Milliseconds; Con, congruent; Inc, incongruent. Error bars are within-subject SEMs.

Post hoc paired $t$ tests for the four stimulation groups were calculated as appropriate. Blinding was tested using binomial and $\chi^{2}$ tests.

\section{Results}

\section{Behavioral results}

Mean RTs of correct responses from all groups and conditions are provided in Table 1. To provide a clearer overview on our data, Figure 1 depicts flanker effects (i.e., the RT difference between incongruent and congruent trials) as a function of the congruency of the previous trial in all groups and stimulation conditions. Below, we additionally report the magnitude of conflict adaptation effects calculated as the flanker effect following congruent trials minus the flanker effect following incongruent trials, which corresponds to the slope of the lines in Figure 1. The five-way ANOVA on mean RTs revealed a significant two-way interaction between Current Congruency and Previous Congruency $\left(F_{(1,116)}=420.1, p<.001\right)$, indicating a robust overall conflict adaptation effect (mean CA $\pm \mathrm{SE}, 20.7 \pm 1.0 \mathrm{~ms}$ ). Cru- 


\begin{tabular}{|c|c|c|c|c|}
\hline & cC & $\mathrm{cl}$ & iC & il \\
\hline \multicolumn{5}{|l|}{ Left DLPFC } \\
\hline Active & $1.8( \pm 0.4)$ & $13.1( \pm 0.9)$ & $1.8( \pm 0.4)$ & $6.4( \pm 0.3$ \\
\hline Sham & $2.0( \pm 0.4)$ & $12.3( \pm 1.0)$ & $2.4( \pm 0.4)$ & $6.4( \pm 0.4$ \\
\hline \multicolumn{5}{|c|}{ Right DLPFC } \\
\hline Active & $1.7( \pm 0.6)$ & $11.4( \pm 0.9)$ & $2.0( \pm 0.6)$ & $7.2( \pm 0.5$ \\
\hline Sham & $1.9( \pm 0.6)$ & $11.9( \pm 1.1)$ & $2.0( \pm 0.6)$ & $7.2( \pm 0.5$ \\
\hline \multicolumn{5}{|l|}{ Left M1 } \\
\hline Active & $1.7( \pm 0.6)$ & $14.4( \pm 1.0)$ & $1.8( \pm 0.5)$ & $7.6( \pm 0.5$ \\
\hline Sham & $1.7( \pm 0.5)$ & $14.4( \pm 0.9)$ & $2.0( \pm 0.4)$ & $7.4( \pm 0.5$ \\
\hline \multicolumn{5}{|l|}{ Right M1 } \\
\hline Active & $1.7( \pm 0.8)$ & $17.8( \pm 1.4)$ & $1.8( \pm 0.8)$ & $10.1( \pm 0.7$ \\
\hline Sham & $1.9( \pm 0.7)$ & $16.6( \pm 1.2)$ & $2.0( \pm 0.7)$ & $9.4( \pm 0.5$ \\
\hline
\end{tabular}

CC, Previous congruent, current congruent; $\mathrm{cl}$, previous congruent, current incongruent; $i C$, previous incongruent, current congruent; il, previous incongruent, current incongruent. Values in brackets are within-subject SEMs.

cially, there was a significant four-way interaction between Region, Stimulation, Current Congruency, and Previous Congruency $\left(F_{(1,116)}=4.56, p<.035\right)$. To elucidate the source of this interaction, we conducted separate ANOVAs for the DLPFC and M1 groups. In the DLPFC groups, a significant three-way interaction between Stimulation, Current Congruency, and Previous Congruency $\left(F_{(1,58)}=9.53, p<.003\right)$ indicated larger conflict adaptation in the active condition $(19.9 \pm 0.8 \mathrm{~ms})$ than in the sham condition $(15.1 \pm 0.8 \mathrm{~ms})$. This effect was not further modulated by Laterality $\left(F_{(1,58)}=0.02, p=0.90\right)$. In the M1 groups, conflict adaptation as reflected by a significant interaction between Current Congruency and Previous Congruency $\left(F_{(1,58)}=\right.$ 257.7, $p<.0001$ ) was not further influenced by stimulation (active, $23.3 \pm 1.2 \mathrm{~ms}$; sham, $\left.24.5 \pm 1.2 \mathrm{~ms} ; F_{(1,58)}=0.26, p=0.61\right)$.

In addition to these effects on conflict adaptation, the five-way ANOVA also revealed a significant interaction between Region and Laterality $\left(F_{(1,116)}=7.09, p<.009\right)$. This reflects that mean RTs in the DLPFC groups were overall higher when stimulation was applied to the right hemisphere (mean RT \pm SE, $307 \pm 9 \mathrm{~ms}$ ) as compared to the left hemisphere $(277 \pm 7 \mathrm{~ms})$, whereas the opposite was obtained for the M1 groups (right M1 group, $284 \pm$ $8 \mathrm{~ms}$; left M1 group, $296 \pm 8 \mathrm{~ms}$ ).

Figure 1 suggests that the effects of active stimulation in the DLPFC groups are not restricted to trials following congruent or incongruent trials. Instead of altering RTs in single data points, active stimulation increased the slopes in Figure 1, and thus conflict adaptation as a whole. To investigate whether the three-way interaction reported for the DLPFC groups can be attributed either to an increased flanker effect following congruent trials or a decreased flanker effect following incongruent trials, we analyzed RTs from the DLPFC groups separately for trials following congruent and incongruent trials. However, the interaction between Current Congruency and Stimulation was not significant in these analyses $(F$ values $<2$ ), indicating that the three-way interaction reported above reflects the combined effect of both levels of previous congruency.

Table 2 shows mean error rates in all groups and conditions. Repeating the five-way ANOVA on error rates again revealed a significant two-way interaction between Current Congruency and Previous Congruency $\left(F_{(1,116)}=384.1, p<.0001\right)$, indicating a robust overall conflict adaptation effect (mean $\mathrm{CA} \pm \mathrm{SE}$, $6.5 \pm 0.4 \%$ ). Furthermore, the effect of Current Congruency was larger in the M1 group (current incongruent minus current con- gruent, $10.4 \pm 0.9 \%)$ than the DLPFC group $(7.52 \pm 0.69 \%$, $\left.F_{(1,116)}=7.69, p<.007\right)$, and the effect of Previous Congruency was larger in the $\mathrm{M} 1$ group (previous congruent minus previous incongruent, $3.54 \pm 0.25 \%)$ than the DLPFC group (2.62 \pm $\left.0.23 \%, F_{(1,116)}=6.67, p<0.02\right)$. Crucially, however, none of these effects involved the variable Stimulation, and therefore were not caused by active stimulation.

In a first control analysis, we reanalyzed the DLPFC groups involving the additional variable Response Repetition (repetition, switch) to investigate whether the effects of active stimulation on conflict adaptation were larger for or were restricted to response repetitions, and thus, rely on priming (Mayr et al., 2003). Indeed, conflict adaptation effects were larger on response repetition trials $(19.2 \pm 1.6 \mathrm{~ms})$ than on response switches $\left(16.2 \pm 1.46 \mathrm{~ms}, F_{(1,58)}=4.31, p<0.05\right)$. However, this effect did not further interact with Stimulation $\left(F_{(1,58)}=0.45, p=0.51\right)$, suggesting that our effect is unrelated to priming. In a second control analysis, we tested whether the effect of stimulation on conflict adaptation significantly changes in the course of the experiment. To this end, the DLPFC groups were analyzed with the additional variable Block (1-5). However, the effect of stimulation on conflict adaptation did not significantly interact with Block, neither in RTs nor in error rates (all $F$ values $<0.8$ ).

Finally, we also explored behavioral effects in both $\mathrm{M} 1$ groups with the additional variable Response Laterality (left vs right hand responses) to investigate effects of M1 stimulation on performance. While the M1 groups were primarily included as a control condition in which no effect of stimulation on conflict adaptation was expected, one could hypothesize that stimulation of this region has an effect on executing responses with the contralateral response hand. However, no significant effects of M1 tDCS on RTs for the stimulated contralateral hand were found (all $p>0.16$ ). For error rates, one would expect that stimulation would lead to increased error rates for trials where the response ipsilateral to stimulation was correct, and thus for which errors occurred with the hand contralateral to stimulation. Indeed, for the right M1 group, the ANOVA revealed a trend toward an interaction between Current Congruency, Stimulation, and Response $\left(F_{(1,29)}=2.98, p<0.10\right)$, indicating that more errors on right-hand trials than on left-hand trials occurred for incongruent trials with active stimulation ( $14.4 \pm 1.1 \%$ vs $13.4 \pm 1.1 \%)$, but not in the other conditions (incongruent vs sham, $13.0 \pm 0.9$ vs $13.0 \pm 1.0 \%$; congruent vs active, $1.4 \pm 0.8$ vs $2.1 \pm 0.8 \%$; congruent vs sham, $1.6 \pm 0.8$ vs $2.2 \pm 0.7 \%)$. No comparable effect was obtained for the left M1 group $(F<0.5)$.

\section{Adverse effects and blinding}

All participants tolerated the stimulation well, and only minor adverse effects were observed (Table 3 ). Adverse effects ratings were not significantly different between the stimulation conditions across the nine adverse effects scales (main effect of Region, all $\left.F_{(3,116)}=0.03-2.17, p=0.08-0.99\right)$. Although mild scalp pain was significantly more pronounced during anodal compared to sham tDCS (Stimulation, $F_{(1,116)}=6.15, p=0.01$ ), the individual post hoc paired $t$ tests for the four stimulation groups did not reach significance $\left(t_{(29)}=0.70-1.72, p=0.09-0.48\right)$. For the remaining eight scales there were no significant effects (all $F=0.6-1.34, p=0.24-0.80)$. None of the interactions between Region and Stimulation reached significance $\left(\right.$ all $F_{(3,116)}=0.06-$ $1.44, p=0.23-0.97)$. The blinded investigator noted only mild skin irritation (maximum score 10 , mean \pm SE, sham tDCS, $1.57 \pm 0.05$; anodal tDCS, $1.69 \pm 0.07$ ). While this mild skin irritation was rated as more pronounced during anodal tDCS 


\begin{tabular}{|c|c|c|c|c|c|}
\hline & Condition & RDLPFC & LDLPFC & RM1 & LM1 \\
\hline \multirow[t]{2}{*}{ Headache } & Active & $1.1( \pm 0.1)$ & $1.2( \pm 0.1)$ & $1.1( \pm 0.0)$ & $1.1( \pm 0.1)$ \\
\hline & Sham & $1.1( \pm 0.1)$ & $1.1( \pm 0.1)$ & $1.2( \pm 0.1)$ & $1.1( \pm 0.1)$ \\
\hline \multirow[t]{2}{*}{ Neck pain } & Active & $1.0( \pm 0.0)$ & $1.1( \pm 0.1)$ & $1.1( \pm 0.1)$ & $1.0( \pm 0.0)$ \\
\hline & Sham & $1.0( \pm 0.0)$ & $1.1( \pm 0.1)$ & $1.2( \pm 0.1)$ & $1.0( \pm 0.0)$ \\
\hline \multirow[t]{2}{*}{ Scalp pain } & Active & $1.1( \pm 0.1)$ & $1.1( \pm 0.1)$ & $1.2( \pm 0.1)$ & $1.2( \pm 0.1)$ \\
\hline & Sham & $1.1( \pm 0.1)$ & $1.1( \pm 0.1)$ & $1.1( \pm 0.1)$ & $1.0( \pm 0.1)$ \\
\hline \multirow[t]{2}{*}{ Tingling } & Active & $1.3( \pm 0.1)$ & $1.5( \pm 0.1)$ & $1.6( \pm 0.1)$ & $1.5( \pm 0.1)$ \\
\hline & Sham & $1.3( \pm 0.1)$ & $1.4( \pm 0.1)$ & $1.4( \pm 0.1)$ & $1.4( \pm 0.1)$ \\
\hline \multirow[t]{2}{*}{ Itching } & Active & $1.2( \pm 0.1)$ & $1.3( \pm 0.1)$ & $1.4( \pm 0.1)$ & $1.4( \pm 0.1)$ \\
\hline & Sham & $1.2( \pm 0.1)$ & $1.2( \pm 0.1)$ & $1.3( \pm 0.1)$ & $1.3( \pm 0.1)$ \\
\hline \multirow[t]{2}{*}{ Burning } & Active & $1.4( \pm 0.1)$ & $1.6( \pm 0.1)$ & $1.4( \pm 0.1)$ & $1.3( \pm 0.1)$ \\
\hline & Sham & $1.4( \pm 0.1)$ & $1.5( \pm 0.1)$ & $1.4( \pm 0.1)$ & $1.2( \pm 0.1)$ \\
\hline \multirow[t]{2}{*}{ Sleepiness } & Active & $2.1( \pm 0.2)$ & $1.6( \pm 0.1)$ & $1.9( \pm 0.1)$ & $2.1( \pm 0.2)$ \\
\hline & Sham & $1.9( \pm 0.2)$ & $1.7( \pm 0.2)$ & $2.1( \pm 0.2)$ & $2.2( \pm 0.2)$ \\
\hline \multirow[t]{2}{*}{ Trouble concentrating } & Active & $1.6( \pm 0.1)$ & $1.5( \pm 0.1)$ & $1.5( \pm 0.1)$ & $1.7( \pm 0.1)$ \\
\hline & Sham & $1.5( \pm 0.1)$ & $1.6( \pm 0.1)$ & $1.7( \pm 0.1)$ & $1.5( \pm 0.1)$ \\
\hline \multirow[t]{2}{*}{ Mood changes } & Active & $1.1( \pm 0.1)$ & $1.0( \pm 0.0)$ & $1.2( \pm 0.1)$ & $1.1( \pm 0.1)$ \\
\hline & Sham & $1.1( \pm 0.1)$ & $1.1( \pm 0.0)$ & $1.2( \pm 0.1)$ & $1.1( \pm 0.1)$ \\
\hline
\end{tabular}

Means and standard error of the mean are reported. $R$, Right; $L$, left.

(Stimulation, $\left.F_{(1,116)}=5.71, p=0.01\right)$, ratings were not different in the four stimulation conditions (Region by Stimulation, $F_{(3}$, $116)=0.66, p=0.57)$. Fifty-seven of the 120 participants guessed the order of the stimulation correctly (right DLPFC, 37\%; left DLPFC, 50\%; right M1, 57\%; left M1, 47\%). The blinded investigator guessed correctly in 46 of 120 instances (right DLPFC, 53\%; left DLPFC, 43\%; right M1, 30\%; left M1, 27\%). Thus, participants and the investigator identified real tDCS approximately at a chance ratio or below in all conditions. Blinding efficacy was comparable in the four groups (participant, $\chi^{2}=2.52$, $p=0.42$; investigator, $\chi^{2}=5.80, p=0.12$ ). In sum, these findings rule out that potential effects of HD-tDCS were mediated by differences in adverse effects or blinding effectiveness.

\section{Discussion}

We provide evidence for a causal role of the DLPFC in adaptive cognitive control as indicated by conflict adaptation. The conflict monitoring theory assumes that the DLPFC implements control adjustments following conflict detection in the medial frontal cortex (Botvinick et al., 2001). Until now, this assumption has predominantly been based on correlative evidence, i.e., on the finding that trials following incongruent flanker stimuli are associated with an increased BOLD response in the DLPFC (Egner and Hirsch, 2005; Egner et al., 2008) and that DLPFC activity in the current trial correlates with ACC activity in the preceding trial (Kerns et al., 2004). In contrast, evidence for a causal involvement of the DLPFC in CA comes only from animal studies (Mansouri et al., 2007). By showing that active HD-tDCS of both the right and left DLPFC enhanced control adjustments, the present study established a direct and causal involvement of those regions in human CA. In contrast, no such enhancement was obtained during stimulation of a nearby control region (M1), demonstrating considerable regional specificity of stimulation effects.

Our results are consistent with the central claim of the conflict monitoring theory, that control is adjusted by shifting attention either toward the target (following incongruent trials) or toward the distractor (following congruent trials). Indeed, the DLPFC has long been assumed to play a key role in biasing spatial attention (Miller and Cohen, 2001; Buschman and Miller, 2007), and CA has been shown to be accompanied by increased activity in target-specific processing areas presumably reflecting increased attention to the target (Egner and Hirsch, 2005). Our results indicate that stronger control adjustments occur due to stimulation of the DLPFC. These stronger control adjustments are not only reflected by a numerically decreased flanker effect following incongruent trials, but similarly by a numerically increased flanker effect following congruent trials. This is reasonable because optimal adaptive control implies not only suppressed distractor processing if distractors are expected to cause conflict (on incongruent trials), but also enhanced distractor processing if distractors are expected to facilitate responding (as on congruent trials).

Although this control account of CA has dominated the literature, alternative theories have been proposed to explain behavioral CA effects (Weissman et al., 2016). Importantly, memory-related mechanisms like feature binding (Hommel et al., 2004) or priming (Mayr et al., 2003) accounted for at least a portion of the CA effect. The feature binding account assumes that CA reflects performance benefits due to full stimulus repetitions on congruent-congruent sequences and incongruent-incongruent sequences. This could potentially explain the present results because a previous study showed increased feature binding effects with DLPFC tDCS (Zmigrod et al., 2014). However, the present effects on CA are more likely to be mediated by control adjustments than feature binding for several reasons. First, previous studies provided evidence that presenting the distractor before the target, as in our study, increases the contribution of control adjustments to the CA effect (Weissman et al., 2014; Weissman et al., 2015). Second, stimulation effects were not stronger for response repetitions (for which stimulus repetitions are possible) than for response switches. Finally, enhanced CA in the present study was found for stimulation of left and right DLPFC, whereas enhanced feature binding was observed only following right DLPFC stimulation (Zmigrod et al., 2014). Nevertheless, the results of the present study alone cannot settle the dispute on the mechanisms underlying CA. However, HD-tDCS might be a promising method to disentangle these accounts in future studies.

Our results further suggest that the contribution of the DLPFC to adaptive cognitive control is not restricted to or stronger in either the left or right hemisphere. Hemispheric asymmetries in DLPFC function have frequently been proposed in the context of cognitive control (Vanderhasselt et al., 2009), but also in other domains like spatial versus verbal working memory or memory encoding versus retrieval (Habib et al., 2003; Wager and Smith, 2003). With respect to adaptive cognitive control, Vanderhasselt et al. (2009) proposed that the left DLPFC is involved when attention has to be adjusted proactively during preparation. In contrast, right DLPFC is recruited when attention is adjusted online and retroactively in response to the detection of conflict. The latter conclusion was based on the finding that right DLPFC is activated during conflict adaptation in the Stroop task (Kerns et al., 2004; Egner et al., 2008). However, other studies using the same task provided evidence for a left DLPFC involvement (MacDonald et al., 2000; Kim et al., 2014) or even bilateral effects (van Veen and Carter, 2005). This heterogeneity as well as the present findings clearly contradict a robust lateralization of control in the DLPFC.

Our study also adds to the growing literature demonstrating beneficial effects of brain stimulation on different aspects of cognitive control, including postconflict and posterror adjustments (Vanderhasselt et al., 2013; Reinhart and Woodman, 2014; Plewnia et al., 2015; Reinhart et al., 2015; Zmigrod et al., 2016). For example, Reinhart and Woodman (2014) demonstrated polarity specific modulation of posterror adjustments by medialfrontal tDCS in healthy individuals. Subsequently, it was shown 
that $\mathrm{tDCS}$ to the same region improved abnormal posterror slowing in patients with schizophrenia to the level of healthy controls and also normalized theta oscillations in the EEG (Reinhart et al., 2015). The latter finding was interpreted as a marker of enhanced functional coordination between brain regions implicated in cognitive control (i.e., medial frontal and DLPFC). Whereas these studies obtained improved cognitive control by stimulating a region involved in the monitoring of errors and conflict, the present study achieved beneficial effects by stimulating a region involved in control adjustment.

Beneficial effects on cognition were often achieved by using conventional tDCS setups, where the current is projected between two fairly large electrodes. Because of the size of the electrodes, such setups result in relatively nonfocal stimulation, and brain areas in between the active and reference electrode may be affected (Kuo et al., 2013; Bortoletto et al., 2016). This complicates the interpretation of the neural mechanisms underlying positive behavioral effects in tDCS studies. In contrast, the present study used a novel HD-tDCS setup that allows current delivery with high spatial precision (Kuo et al., 2013; Villamar et al., 2013; Alam et al., 2016). This setup has been shown to result in more pronounced behavioral or neurophysiological effects than conventional tDCS (Kuo et al., 2013; Nikolin et al., 2015; Richardson et al., 2015; Gbadeyan et al., 2016). While this study did not directly compare conventional and high-definition tDCS, the positive effects on CA suggest that this novel type of tDCS is effective to modulate cognition in a regionally specific way. Moreover, to the best of our knowledge, this is the first study that demonstrates regionally specific behavioral modulation of cognition by HD-tDCS across multiple stimulation sites.

Some limitations need to be acknowledged. Previous modeling studies have suggested more focal current delivery by HD versus conventional tDCS (Kuo et al., 2013; Bortoletto et al., 2016). However, selective neural modulation of the DLPFC needs to be verified in future studies combining HD-tDCS with functional imaging. This would also allow verifying the location of the electrodes with regard to the target regions (Ulm et al., 2015). Despite substantial modulation of RTs after DLPFC HD-tDCS, no effects were found for error rates. This is likely explained by low error rates in our healthy young sample. Accordingly, errors were mainly analyzed to exclude that speed-accuracy trade-offs underlie our RT effects, which was not the case. Moreover, due to the between-group design, group differences in mean RTs were larger than tDCS-induced effects in some groups. However, these differences were similar during active and sham tDCS and cannot reflect stimulation effects. Moreover, these effects cannot account for different effects of active stimulation in our groups, because mean RT differences do not mirror stimulation effects on CA. Please note, a five-way crossover design would likely have reduced feasibility (i.e., increased dropout rates) and induced other confounding factors (e.g., motivational issues or practice effects).

\section{Conclusions}

Enhanced CA after DLPFC HD-tDCS demonstrates that this region is causally involved in adaptive cognitive control, thereby supporting a central claim of the conflict monitoring theory (Botvinick et al., 2001). We also provide strong evidence that both the left and right DLPFC are involved in this essential human function, and we assured regional specificity of the stimulation effects by including two additional active stimulation sites that did not modulate CA. Aside from theoretical implications for current theories of cognitive control, our results provide a rationale to explore potential beneficial effects of DLPFC HDtDCS in clinical populations with impaired cognitive control. This is suggested by the considerable size of stimulation effects. Although effects on absolute RTs were small, DLPFC stimulation increased CA by $>30 \%$ relative to sham stimulation. These positive results lay the ground to explore beneficial effects of HDtDCS in ecologically relevant experimental contexts and in patient populations with impaired CA ability. Moreover, we demonstrated previously the compatibility of HD-tDCS with fMRI (Gbadeyan et al., 2016), which will allow us and others to scrutinize the neural mechanisms underlying those beneficial behavioral effects in future studies.

\section{References}

Alam M, Truong DQ, Khadka N, Bikson M (2016) Spatial and polarity precision of concentric high-definition transcranial direct current stimulation (HD-tDCS). Phys Med Biol 61:4506-4521. CrossRef Medline

Bortoletto M, Rodella C, Salvador R, Miranda PC, Miniussi C (2016) Reduced current spread by concentric electrodes in transcranial electrical stimulation (tES). Brain Stimul 9:525-528. CrossRef Medline

Botvinick MM, Braver TS, Barch DM, Carter CS, Cohen JD (2001) Conflict monitoring and cognitive control. Psychol Rev 108:624-652. CrossRef Medline

Brunoni AR, Amadera J, Berbel B, Volz MS, Rizzerio BG, Fregni F (2011) A systematic review on reporting and assessment of adverse effects associated with transcranial direct current stimulation. Int J Neuropsychopharmacol 14:1133-1145. CrossRef Medline

Buschman TJ, Miller EK (2007) Top-down versus bottom-up control of attention in the prefrontal and posterior parietal cortices. Science 315: 1860-1862. CrossRef Medline

Coffman BA, Clark VP, Parasuraman R (2014) Battery powered thought: Enhancement of attention, learning, and memory in healthy adults using transcranial direct current stimulation. Neuroimage 85:895-908. CrossRef Medline

Cousineau D (2005) Confidence intervals in within-subject designs: a simpler solution to Loftus and Masson's method. Tutor Quant Methods Psychol 1:42-45. CrossRef

Dedoncker J, Brunoni AR, Baeken C, Vanderhasselt MA (2016) A systematic review and meta-analysis of the effects of transcranial direct current stimulation (tDCS) over the dorsolateral prefrontal cortex in healthy and neuropsychiatric samples: influence of stimulation parameters. Brain Stimul 9:501-517. CrossRef Medline

Egner T, Hirsch J (2005) Cognitive control mechanisms resolve conflict through cortical amplification of task-relevant information. Nat Neurosci 8:1784-1790. CrossRef Medline

Egner T, Etkin A, Gale S, Hirsch J (2008) Dissociable neural systems resolve conflict from emotional versus nonemotional distracters. Cereb Cortex 18:1475-1484. CrossRef Medline

Eriksen B, Eriksen C (1974) Effects of noise letters upon the identification of a target letter in a nonsearch task. Percept Psychophys 16:143-149. CrossRef

Gbadeyan O, Steinhauser M, McMahon K, Meinzer M (2016) Safety, tolerability, blinding efficacy and behavioural effects of a novel MRIcompatible, high-definition tDCS set-up. Brain Stimul 9:545-552. CrossRef Medline

Gratton G, Coles MG, Donchin E (1992) Optimizing the use of information: Strategic control of activation of responses. J Exp Psychol Gen 121: 480-506. CrossRef Medline

Habib R, Nyberg L, Tulving E (2003) Hemispheric asymmetries of memory: the HERA model revisited. Trends Cogn Sci 7:241-245. CrossRef Medline

Hogeveen J, Grafman J, Aboseria M, David A, Bikson M, Hauner KK (2016) Effects of high-definition and conventional tDCS on response inhibition. Brain Stimul 9:720-729. CrossRef Medline

Hommel B, Proctor RW, Vu KP (2004) A feature-integration account of sequential effects in the Simon task. Psychol Res 68:1-17. CrossRef Medline

Kerns JG, Cohen JD, MacDonald AW 3rd, Cho RY, Stenger VA, Carter CS (2004) Anterior cingulate conflict monitoring and adjustments in control. Science 303:1023-1026. CrossRef Medline

Kim C, Johnson NF, Gold BT (2014) Conflict adaptation in prefrontal cortex: now you see it, now you don't. Cortex 50:76-85. CrossRef Medline 
Kuo HI, Bikson M, Datta A, Minhas P, Paulus W, Kuo MF, Nitsche MA (2013) Comparing cortical plasticity induced by conventional and highdefinition $4 \times 1$ ring tDCS: a neurophysiological study. Brain Stimul 6:644-648. CrossRef Medline

MacDonald AW 3rd, Cohen JD, Stenger VA, Carter CS (2000) Dissociating the role of the dorsolateral prefrontal and anterior cingulate cortex in cognitive control. Science 288:1835-1838. CrossRef Medline

Mansouri FA, Buckley MJ, Tanaka K (2007) Mnemonic function of the dorsolateral prefrontal cortex in conflict-induced behavioral adjustment. Science 318:987-990. CrossRef Medline

Mansouri FA, Tanaka K, Buckley MJ (2009) Conflict-induced behavioural adjustment: a clue to the executive functions of the prefrontal cortex. Nat Rev Neurosci 10:141-152. Medline

Mansouri FA, Fehring DJ, Feizpour A, Gaillard A, Rosa MG, Rajan R, Jaberzadeh S (2016) Direct current stimulation of prefrontal cortex modulates error-induced behavioral adjustments. Eur J Neurosci 44:18561869. CrossRef Medline

Mayr U, Awh E, Laurey P (2003) Conflict adaptation effects in the absence of executive control. Nat Neurosci 6:450-452. Medline

Miller EK (2000) The prefontral cortex and cognitive control. Nat Rev Neurosci 1:59-65. CrossRef Medline

Miller EK, Cohen JD (2001) An integrative theory of prefrontal cortex function. Annu Rev Neurosci 24:167-202. CrossRef Medline

Nikolin S, Loo CK, Bai S, Dokos S, Martin DM (2015) Focalised stimulation using high definition transcranial direct current stimulation (HD-tDCS) to investigate declarative verbal learning and memory functioning. Neuroimage 117:11-19. CrossRef Medline

Plewnia C, Schroeder PA, Kunze R, Faehling F, Wolkenstein L (2015) Keep calm and carry on: improved frustration tolerance and processing speed by transcranial direct current stimulation (tDCS). PLoS One 10: e0122578. CrossRef Medline

Reinhart RM, Woodman GF (2014) Causal control of medial-frontal cortex governs electrophysiological and behavioral indices of performance monitoring and learning. J Neurosci 34:4214-4227. CrossRef Medline

Reinhart RM, Zhu J, Park S, Woodman GF (2015) Synchronizing theta oscillations with direct-current stimulation strengthens adaptive control in the human brain. Proc Natl Acad Sci U S A 112:9448-9453. CrossRef Medline

Richardson J, Datta A, Dmochowski J, Parra LC, Fridriksson J (2015) Feasibility of using high-definition transcranial direct current stimulation (HD-tDCS) to enhance treatment outcomes in persons with aphasia. NeuroRehabilitation 36:115-126. Medline
Stagg CJ, Nitsche MA (2011) Physiological basis of transcranial direct current stimulation. Neuroscientist 17:37-53. CrossRef Medline

Ulm L, McMahon K, Copland D, de Zubicaray GI, Meinzer M (2015) Neural mechanisms underlying perilesional transcranial direct current stimulation in aphasia: a feasibility study. Front Hum Neurosci 9:550. Medline

Vanderhasselt MA, De Raedt R, Baeken C (2009) Dorsolateral prefrontal cortex and Stroop performance: tackling the lateralization. Psychonom Bull Rev 16:609-612. CrossRef

Vanderhasselt MA, De Raedt R, Brunoni AR, Campanhã C, Baeken C, Remue J, Boggio PS (2013) tDCS over the left prefrontal cortex enhances cognitive control for positive affective stimuli. PLoS One 8:e62219. CrossRef Medline

van Veen V, Carter CS (2005) Separating semantic conflict and response conflict in the Stroop task: a functional MRI study. Neuroimage 27:497504. CrossRef Medline

Villamar MF, Wivatvongvana P, Patumanond J, Bikson M, Truong DQ, Datta A, Fregni F (2013) Focal modulation of the primary motor cortex in fibromyalgia using $4 \times 1$-ring high-definition transcranial direct current stimulation (HD-tDCS): immediate and delayed analgesic effects of cathodal and anodal stimulation. J Pain 14:371-383. CrossRef Medline

Wager TD, Smith EE (2003) Neuroimaging studies of working memory. Cogn Affect Behav Neurosci 3:255-274. CrossRef Medline

Weissman DH, Jiang J, Egner T (2014) Determinants of congruency sequence effects without learning and memory confounds. J Exp Psychol Hum Percept Perform 40:2022-2037. CrossRef Medline

Weissman DH, Egner T, Hawks Z, Link J (2015) The congruency sequence effect emerges when the distracter precedes the target. Acta Psychol 156: 8-21. CrossRef

Weissman DH, Hawks ZW, Egner T (2016) Different levels of learning interact to shape the congruency sequence effect. J Exp Psychol Learn Mem Cogn 42:566-583. CrossRef Medline

Winer B, Brown D, Michels K (1991) Statistical principles in experimental design, Ed 3. New York: McGraw-Hill.

Zmigrod S, Colzato LS, Hommel B (2014) Evidence for a role of the right dorsolateral prefrontal cortex in controlling stimulus-response integration: a transcranial direct current stimulation (tDCS) study. Brain Stimul 7:516-520. CrossRef Medline

Zmigrod S, Zmigrod L, Hommel B (2016) Transcranial direct current stimulation (tDCS) over the right dorsolateral prefrontal cortex affects stimulus conflict but not response conflict. Neuroscience 322:320-325. CrossRef Medline 